\title{
Nuclear Demagnetization Stages for High Magnetic Fields
}

Cooling to very low temperatures by adiabatic demagnetization of a nuclear paramagnet generally involves pre-cooling a copper stage coupled to the sample platform to about $10 \mathrm{mK}$ in a magnetic field of 8-10 T using a powerful dilution refrigerator. A superconducting heat switch then thermally isolates the stage from the mixing chamber of the refrigerator, and the magnetic field is slowly reduced.

The drawing shows a convenient way of coupling a copper demagnetization stage to a powerful dilution refrigerator so as to minimise the distance between the dilution refrigerator and the centre of the measuring field. The demagnetization magnet is in a vacuum, attached to a still, surrounded by heat exchangers. A simple extension of the silver cold finger is used for experiments in very high magnetic fields (usually persistent-mode superconducting magnets).

Eddy current heating is more serious in the hybrid magnets (a conventional outer electromagnet and a superconducting inner magnet) which provide the highest steady-state fields available today. In this case, one could use a plastic dilution refrigerator to pre-cool, to 20-25 mK, a convenient nuclear paramagnet with a large thermal capacity placed at the centre of the magnetic field. The dilution refrigerator would then be withdrawn by about $300 \mathrm{~mm}$ until the demagnetization stage enters a region of $\approx 1 \mathrm{~T}$ at $1.0-1.5 \mathrm{mK}$, while the measuring cell finds itself at the centre of the field. A silver heat exchanger attached to the nuclear stage would be at about the same temperature, and the minimum temperature of the sample depends upon the heat leak and the exchanger area.

Niobium seems the best choice for the stage as calculations show that it has a large entropy reduction per unit volume at $25 \mathrm{~T}$
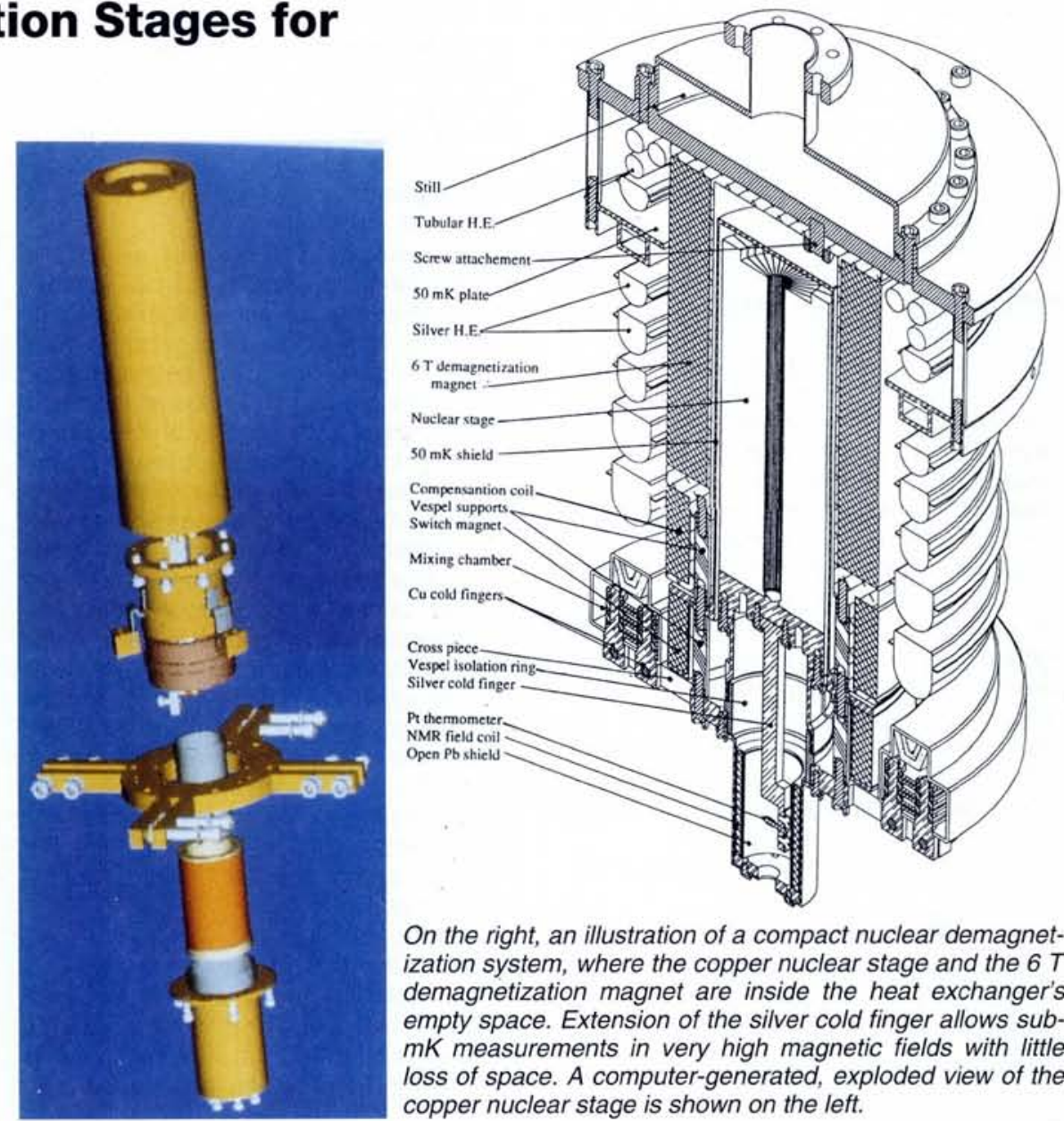

On the right, an illustration of a compact nuclear demagnetization system, where the copper nuclear stage and the $6 T$ demagnetization magnet are inside the heat exchanger's empty space. Extension of the silver cold finger allows sub$m K$ measurements in very high magnetic fields with little loss of space. A computer-generated, exploded view of the copper nuclear stage is shown on the left.

(the final field must be kept above $1 \mathrm{~T}$ to avoid flux jumps as the $\mathrm{Nb}$ is superconducting with a critical field as large as 0.8-0.9 T owing to impurities); aluminium is also a good choice. A heat switch, probably mechanical, needs developing as the usual superconduct- ing switch will not work. The main limitations comes from the silver heat exchanger inside the mixing chamber as it determines the precooling time, a very important consideration in experiments involving $10 \mathrm{MW}$ and larger magnets.

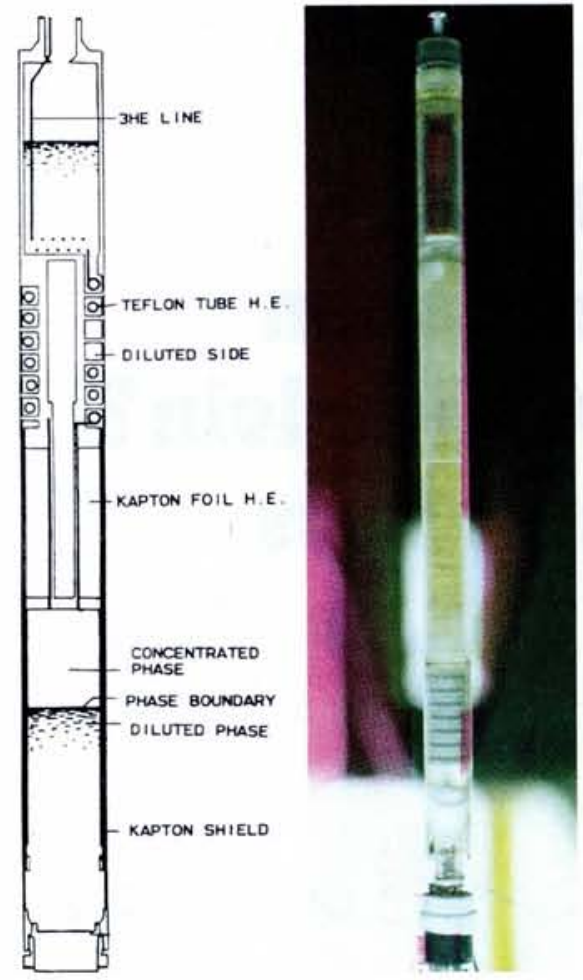

rization, seem to support the paramagnetic model [8]. Experiments at higher fields and stronger polarizations would allow several properties (including $T_{\mathrm{c}}$ ) to be measured to much higher polarizations for a better comparison with the model's predictions.

Since the entropy of the solid is expected to fall below that of the liquid at $8-10 \mathrm{mK}$, there will be the unique opportunity, impossible to realise except with ultra-high fields, of using the melting of ${ }^{3} \mathrm{He}$ below $300 \mathrm{mK}$ to produce cooling. One could reach a regime with a non-exponential $t_{1}$, something that has not yet been achieved, where the liquid's stored magnetic energy exceeds its thermal energy. Recently, we have been able to polarize ${ }^{3} \mathrm{He}-{ }^{4} \mathrm{He}$ mixtures with a high concentration of ${ }^{3} \mathrm{He}$ (a $5.5 \%$ mixture of ${ }^{3} \mathrm{He}$ in ${ }^{4} \mathrm{He}$ gave $25 \%$ polarization with a $t_{1}$

Fig. 2 - A photograph and a schematic illustration of a removable dilution refrigerator insert with a plastic dilution refrigerator for use in very high magnetic fields. The design allows for very large flow rates and cooling power in a small overall diameter (cooling power of $1 \mathrm{~mW}$ at $100 \mathrm{mK}$ with a minimum temperature of $8 \mathrm{mK}[14])$. Courtesy of Leiden Cryogenics b.v. of $4000 \mathrm{~s}$ at $300 \mathrm{mK}$ [9]). Such experiments use the same techniques as for pure ${ }^{3} \mathrm{He}$ and could benefit greatly from higher initial fields giving lower final temperatures, larger polarizations, and longer $t_{1}$. This is probably also true for the method based on fractional distillation of ${ }^{3} \mathrm{He}-{ }^{4} \mathrm{He}$ mixtures [10].

\section{Polarized $\mathrm{D}_{2}$}

Mixtures of polarized ${ }^{3} \mathrm{He}$ and $\mathrm{D}_{2}$ have been proposed as an aneutronic fuel for fusion reactors since the cross-section is $50 \%$ larger than in the unpolarized form (the $\mathrm{D}_{2}$ could be mined on the Moon [11]). Polarized ${ }^{3} \mathrm{He}$ can be produced in large amounts using a Pomeranchuk cell (a cell with flexible walls used to solidify ${ }^{3} \mathrm{He}$ by compression with liquid ${ }^{4} \mathrm{He}$ ) since the polarization remains in the liquid after melting. Laser methods are also used to achieve large polarizations in gases [12].

$D_{2}$ is much more difficult to polarize to the ortho state owing to a smaller magnetic moment and a low thermal diffusivity of the crystal. We have recently polarized $D_{2}$ to $12 \%$ using a novel technique whereby gaseous $D_{2}$ is condensed as small droplets in liquid ${ }^{3} \mathrm{He}$ using a Pomeranchuk cell at 14.5 T [13]. By increasing the field to $30 \mathrm{~T}$ 
and reducing the temperature of the $D_{2}$ from $35 \mathrm{mK}$ to $8 \mathrm{mK}$ one should reach full polarization. The main research challenge will be to remove the polarized ${ }^{3} \mathrm{He}$ and $\mathrm{D}_{2}$ from the cryogenic environment so that depolarization effects at very high temperatures can be studied to see if the polarization can be kept for long enough to be useful in a fusion reactor.

\section{Refrigerators for High Magnetic Fields}

The dilution refrigerator is nowadays the only means of cooling a sample continuously to a few $\mathrm{mK}$, and adiabatic demagnetization of a nuclear paramagnet (see page 109) is needed to reach the lowest temperatures. Dilution refrigerators are based on the expansion of low-entropy ${ }^{3} \mathrm{He}$ into high-entropy ${ }^{3} \mathrm{He}$ diluted in ${ }^{4} \mathrm{He}$. The equilibrium concentration of ${ }^{4} \mathrm{He}$ in ${ }^{3} \mathrm{He}$ at $0 \mathrm{~K}$ is essentially zero while that of ${ }^{3} \mathrm{He}$ diluted in ${ }^{4} \mathrm{He}$ is about $6.5 \%$. If one tries to decrease, by pumping, the limiting concentration of ${ }^{3} \mathrm{He}$ in ${ }^{4} \mathrm{He}$ at a given temperature in the mixing chamber at a temperature $T_{\mathrm{mc}}$ where concentrated ${ }^{3} \mathrm{He}$ is in equilibrium with diluted ${ }^{3} \mathrm{He}$, pure ${ }^{3} \mathrm{He}$ will cross the boundaries and re-establish the equilibrium concentration, thus absorbing heat.

An enthalpy balance at the mixing chamber gives:

$$
\dot{Q} / \dot{n}=94.5 T_{\mathrm{mc}}^{2}-12.5 T_{\mathrm{cp}}^{2}
$$

for a cooling power $\dot{Q}$ in W, circulation rate $\dot{n}$ in $\mathrm{mol} / \mathrm{s}$, temperature $T_{\mathrm{cp}}$ (in degrees kelvin) of the concentrated phase entering the mixing chamber. The maximum cooling power is for $T_{c p}=T_{m c}$, which is attainable with an infinitely large heat exchanger to transfer all the enthalpy of the incoming ${ }^{3} \mathrm{He}$ to the outgoing diluted ${ }^{3} \mathrm{He}$. Heat exchange is usually via a solid separating wall using phonon exchange. Owing to the large difference in the velocity of sound between a solid (generally a metal) and helium, most of the phonons are reflected giving rise to the well-known Kapitza resistance which increases as $T^{-3}$. In practice, the maximum cooling power is obtained with a heat exchanger with a very large area made of a convenient material such as sub-micron silver powder.

\section{Limitations}

For a perfect, continuous heat exchanger, where perfect effectively means that the heat exchanged through the wall is much larger than that generated by viscous heating or brought by conduction to the mixing chamber,

$$
T_{\mathrm{mc}}^{2}=6.5 R_{\mathrm{Km}} \dot{n} / A+0.012 \dot{Q} / \dot{n}
$$

$A$ is the area (in $\mathrm{m}^{2}$ ) of one side of the heat exchanger; $R_{\mathrm{Km}}$ is the average Kapitza resistance in $\mathrm{K}^{4} \mathrm{~m}^{2} / \mathrm{W}$ between helium and the wall of the heat exchanger, and is taken to be $2 R_{K}$ where $R_{K}$ is the Kapitza resistance between diluted ${ }^{3} \mathrm{He}$ and the wall. For fine silver powder, $R_{\mathrm{K}}$ in fact varies as $T^{-2}$ below $10-15 \mathrm{mK}$ so Eq. 2 should be somewhat modified. For very large $\dot{n} / A, T_{\mathrm{mc}}$ is dominated by $\dot{Q} / \dot{n}$, which can be made arbitrarily small for a given input by choosing a large $\dot{n}$.

$\dot{Q}$ is the sum of an external heat leak and an internal, flow-dependent heat leak. For a typical $\dot{Q}$ of $\approx 1 \mu \mathrm{W}$, a circulation rate $\dot{n}=$
$10^{-3} \mathrm{~mol} / \mathrm{s}$ gives a limit to $T_{\mathrm{mc}}$ of $3.16 \mathrm{mK}$ for an infinite area of heat exchanger. However temperatures below $3 \mathrm{mK}$ and $\dot{n}$ of the order $10^{-3} \mathrm{~mol} / \mathrm{s}$ require flow channels on the diluted side of the exchanger of $10-20 \mathrm{~mm}$ in diameter, so continuous temperatures of this magnitude are effectively excluded for small-bore magnets.

Since viscous heating varies as $T^{-2}$ and thermal conduction as $T^{-1}$, a small increase in $T_{\mathrm{mc}}$ allows the channel diameter to be decreased to a manageable size. Thus, at $10 \mathrm{mK}$, the flow-dependent heat leak is no longer a major problem and careful attention must be given to the design of the installation to minimise the flow-independent, external heat leak.

Having chosen the heat exchanger material (and hence $R_{\mathrm{Km}}$ ) and $\dot{n}$, we are left with maximising the heat exchanger area $A$. For sintered, porous $\mathrm{Ag}$ sponge, the most efficient material in low magnetic fields, about $2 \mathrm{~kg}$ of powder with a $50 \%$ filling factor was used to attain $1.9 \mathrm{mK}$ at $\dot{n}=10^{-3} \mathrm{~mol} / \mathrm{s}$. Such refrigerators are too large to be placed inside, or even close to, ultra-high magnetic fields. The usual technique therefore involves locating the refrigerator outside the magnet and having a long cold finger or a plastic mixing chamber extend into the high-field region.

\section{Plastic dilution refrigerators}

The velocity of sound and the density of plastic are much smaller than for a metal, so $R_{K}$ is smaller. If the heat exchanger wall is thin enough (tens of $\mu \mathrm{m}$ ), thermal conduction across the foil below $100 \mathrm{mK}$ will be significantly larger than the Kapitza conduction. Plastic then becomes superior to metal as a heat exchanger material. The area per unit volume of the plastic exchanger will of course be smaller than for sintered $\mathrm{Ag}$, but the thermal resistance is about the same for plastic and $\mathrm{Ag}$ heat exchangers of a given volume at $25 \mathrm{mK}$, the plastic device being superior at higher temperatures. Owing to the absence of eddy currents, plastic exchangers are in fact also superior at lower temperatures. Using a kapton foil heat exchanger with areas up to $0.12 \mathrm{~m}^{2}$, we have developed plastic dilution refrigerators of diameters between 14 and $40 \mathrm{~mm}$ that reach temperatures below $8 \mathrm{mK}$ (Fig. 2) in very high magnetic fields. By raising $B / T$ ratios, these devices, along with advanced demagnetization stages, should allow us to continue to push back the frontiers of solid-state physics.

[1] Halperin W.P., et al., Phys. Rev. Lett. 32 (1974) 927; Kummer R.B., et al., ibid. 34 (1975) 517.

[2] Osheroff D., Cross M.C. \& Fisher D.S., ibid. 44 (1980) 792; Prewitt T.C. \& Goodkind J.M., ibid. 39 (1977) 1283.

[3] Roger M., Hetherington J.H. \& Delrieu J.M., Rev. Mod. Phys. 55 (1983) 1; Kummer R.B., Müller R.M. \& Adams E.D., J. Low Temp. Phys. 27 (319) (1977) 319

[4] Kranenburg C.C., et al., Jpn. J. Appl. Phys. Supp 26-3 26 (1987) 2133; Frossati G., et al., Czech. J. Phys. 40 (1990) 909.

[5] Osheroff D.D., Godfrin H. \& Ruel R., Phys. Rev. B 36 (1987) 6853; Godfrin H. \& Osheroff D.D., ibid. 38 (1988) 4492.

[6] Wiegers S.A.J., et al., Physica B 165-166 (1990) 733.

[7] Bedell K.S. \& Sanchez-Castro C., Phys. Rev. Lett. 57 (1986) 854

[8] Béal-Monod M.T. \& Daniel E., ibid., 27 (1983) 4467.

[9] Roobol L.P., et al., Europhys. Lett. 17 (1992) 219.

[10] Nacher P.J., et al., Phys. Rev. Lett. 67 (1991) 839; Vermeulen G.A., et al. (see Monceau P., page 94).

[11] Kulcinski G.L., et al., Fusion Tech. 15 (1989) 1233.

[12] Himbert M., et al., J. Phys. Lett. (Paris) 46 (1985) 249.

[13] Ter Haar E., Clark W.G. \& Frossati G. Proc. 20th Conf. on Low-Temperature Physics, Eugene, USA (to be published).

[14] Wagner R. \& Frossati G., Physica B 165$166(1990) 43$
From ultra-low temperature measurement and HTc superconductors through the latest in magnetic measurements and on to high-temperature thermal analysis, Cryophysics can help with your instrumentation problems. Contact René Koch in Geneva for details. 